\title{
Retrieval of cavity-generated atomic spin squeezing after free-space release
}

\author{
Yunfan Wu $\odot,{ }^{1}$ Rajiv Krishnakumar $\odot,{ }^{1,2}$ Julián Martínez-Rincón $\odot,{ }^{3}$ Benjamin K. Malia $\odot,{ }^{3}$ \\ Onur Hosten $\odot,{ }^{4}$ and Mark A. Kasevich ${ }^{1,3,{ }^{*}}$ \\ ${ }^{1}$ Department of Applied Physics, Stanford University, Stanford, California 94305, USA \\ ${ }^{2}$ Department of Physics, PMA, California Institute of Technology, California 91125, USA \\ ${ }^{3}$ Physics Department, Stanford University, Stanford, California 94305, USA \\ ${ }^{4}$ Institute of Science and Technology Austria, Klosterneuburg 3400, Austria
}

(Received 14 December 2019; accepted 15 June 2020; published 30 July 2020)

\begin{abstract}
We demonstrate that releasing atoms into free space from an optical lattice does not deteriorate cavitygenerated spin squeezing for metrological purposes. In this work, an ensemble of 500000 spin-squeezed atoms in a high-finesse optical cavity with near-uniform atom-cavity coupling is prepared, released into free space, recaptured in the cavity, and probed. Up to $\sim 10 \mathrm{~dB}$ of metrologically relevant squeezing is retrieved for $700 \mu \mathrm{s}$ free-fall times, and decaying levels of squeezing are realized for up to $3 \mathrm{~ms}$ free-fall times. The degradation of squeezing results from loss of atom-cavity coupling homogeneity between the initial squeezed state generation and final collective state readout. A theoretical model is developed to quantify this degradation and this model is experimentally validated.
\end{abstract}

DOI: 10.1103/PhysRevA.102.012224

\section{INTRODUCTION}

Atomic sensors, including atom interferometers and atomic clocks, typically operate near the quantum projection noise limit permitted by uncorrelated ensembles of atoms [1-4]. This limit can be overcome using quantum entanglement. For example, with spin-squeezed states [5] it is feasible to surpass the performance of current state-of-the-art sensors as long as typical atom numbers can be preserved and large levels of squeezing can be obtained. Experimentally, spin squeezing has been demonstrated through a number of methods [6-14]. To date, the best levels of metrologically relevant squeezing $(\sim 20 \mathrm{~dB})$ have been obtained in systems where cold atoms are trapped and coupled to an optical cavity and the collective state of the atoms is probed through a cavity mode $[6,14]$. In these systems, up to order of one-million atoms can be utilized, conforming to standards of well engineered sensors.

Implementation of squeezed-state protocols in sensors with freely moving atoms-devoid of perturbations due to an external confining potential-requires that the initial squeezed states are prepared in a spatially homogeneous way, i.e., that each atomic spin must contribute equally to the collective spin that is being measured. Otherwise, the retrieval of squeezing is hindered: once the atoms are free to move, the information about their individual contributions to the original collective spin is lost, and a different collective spin, which is not necessarily squeezed, is probed [15]. Methods to meet the homogeneity requirement in cavity-generated spin squeezing experiments have been studied in Refs. [6,16].

Here we show that, by using an optical cavity apparatus specifically designed to enforce homogeneous atom-cavity

\footnotetext{
*kasevich@stanford.edu
}

coupling [6], squeezing can be generated and retrieved after the atoms are released to millisecond-long duration free falls and recaptured back into the cavity. With this configuration, we experimentally characterize the change in atomcavity coupling and its effect on retrieval of spin squeezing. We develop and experimentally validate a theoretical model which quantifies the degradation of squeezing in terms of experimentally accessible observables. Prior work has quantified squeezing for inhomogeneously coupled ensembles where the inhomogeneous coupling is fixed for each atom during both the squeezing and retrieval measurement sequences [9,17]. In this work, we quantitatively assess the impact of changes in coupling homogeneity between the squeezing and retrieval operations. This is relevant to atomic sensors which seek to exploit squeezing for enhanced noise performance.

\section{EXPERIMENTAL SYSTEM}

In our experimental demonstration, we trap $N \sim 500000$ ${ }^{87} \mathrm{Rb}$ atoms inside of a 10-cm-long high-finesse dualwavelength near confocal cavity that supports both $1560 \mathrm{~nm}$ and $780 \mathrm{~nm}$ modes. The $1560 \mathrm{~nm}$ light forms a $520 \mu \mathrm{K}$ deep one dimensional (1D) optical dipole lattice to trap the $25 \mu \mathrm{K}$ atoms, and the $780 \mathrm{~nm}$ light interacts near resonantly with the $D_{2}$ transition of the atoms to act as a dispersive probe $[6,18]$. By design, the trapping locations of atoms are aligned with the intensity maxima of the probe standing wave near the center of the cavity spanning a thousand lattice sites. This alignment gives an almost uniform atom-cavity coupling [Fig. 1(a)]. The magnetically insensitive hyperfine ground states $\left|F=2, m_{F}=0\right\rangle$ and $\left|F=1, m_{F}=0\right\rangle$ constitute the $|\uparrow\rangle$ and $|\downarrow\rangle$ states for a pseudo-spin-1/2 system.

The atom-cavity detuning is set such that, when interacting with the atoms in the $|\uparrow\rangle$ state, the cavity resonance 
(a)

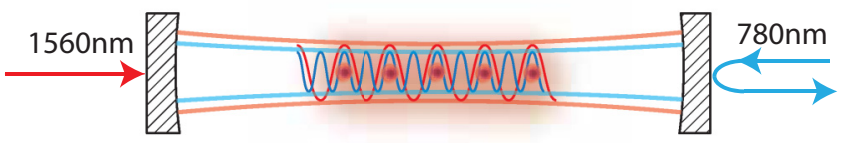

(b)

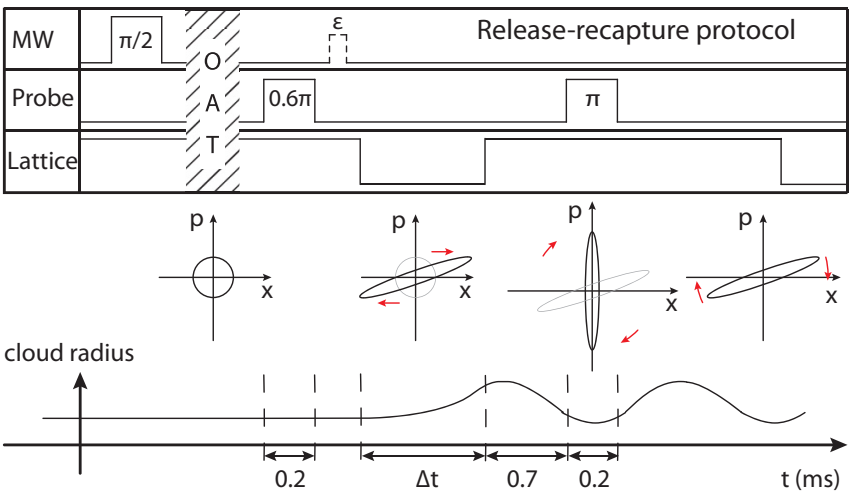

FIG. 1. (a) Near-homogeneous atom-cavity coupling of ${ }^{87} \mathrm{Rb}$ atoms to a $780 \mathrm{~nm}$ probe via a commensurate $1560 \mathrm{~nm}$ optical lattice. (b) Release-recapture protocol: timing sequence, illustrations for phase-space evolution of the atomic cloud in the transverse direction of the cavity, and rms transverse cloud size evolution. In the phase-space illustration, clouds are modeled to execute harmonic oscillations when trapped in the lattice. MW: microwaves; OAT: one-axis twisting; $\pi / 2$ : composite microwave pulse that prepares the initial superposition state; $0.6 \pi$ and $\pi$ : probe power expressed in terms of the relative ac Stark phase shifts induced between the two atomic states; $\Delta t$ : free-fall time; $\epsilon$ : a small microwave rotation.

frequency acquires a shift equal in magnitude but opposite in sign compared to when it interacts with the $|\downarrow\rangle$ state atoms. Measurement of this frequency shift by interferometric monitoring of the light reflected from the cavity realizes a quantum nondemolition (QND) measurement of the collective spin $J_{z}=\sum_{i=1}^{N} j_{z}^{(i)}$, where $j_{z}^{(i)}=\left(|\uparrow\rangle_{i}\left\langle\left.\uparrow\right|_{i}-\mid \downarrow\right\rangle_{i}\left\langle\left.\downarrow\right|_{i}\right) / 2\right.$ refers to the $z$ component of the pseudospin associated with the $i$ th atom [19]. In our work, a single spin flip between the $|\uparrow\rangle$ and $|\downarrow\rangle$ states results in an $\sim 5.6 \mathrm{~Hz}$ shift in the cavity resonance frequency, as determined from our cavity parameters and independently verified by measuring the quantum projection noise for coherent spin states [6].

For the generation of spin squeezed states, we follow the procedure in [6] with an added step of a free space release recapture of the atoms between the preparation and readout probes. A one-axis twisting squeezing procedure [20,21] is performed before the first QND measurement (preparation probe) to allow for squeezing of a large number of atoms [6]. After release and recapture, a second QND measurement (readout probe) is performed to observe the collective spin. The preparation probe is chosen to be weaker than the readout probe to obtain large state coherence. In this work, the maximum recovered metrologically relevant squeezing of this back-to-back measurement protocol is limited to $\sim 13 \mathrm{~dB}$ for such a configuration in the absence of release.

\section{MEASUREMENT PROTOCOL}

The release-recapture protocol is shown in Fig. 1(b). The trapping lattice is turned off after the first probe to release the spin-squeezed atoms in free space. The lattice switching time is $50 \mu \mathrm{s}$, which is adiabatic for the motion in the longitudinal trapping direction but sudden for the transverse one. After a variable free-fall time $\Delta t$ accompanied by a ballistic expansion ( $\sim 5 \mathrm{~cm} / \mathrm{s}$ from a $17 \mu \mathrm{m}$ rms radius), the lattice is turned on again with the same switching time as the release stage to recapture the atoms. After the recapture, the atomic cloud size and position starts oscillating in the transverse direction of the 1D lattice. The maximum recovered squeezing is achieved when the readout probe is turned on while the atomic cloud is maximally compressed during such oscillations.

The ballistic expansion of the atom cloud and acceleration due to gravity lead to an asymmetry in the atom-cavity coupling between the two probes. This asymmetry between the two probes grows with the free-fall time due to the (fixed) Gaussian spatial profile of the cavity mode. Therefore, the readout probe measures a different observable than the preparation probe that degrades the observed squeezing. In principle one can engineer more advanced release-recapture sequences to better preserve the symmetry in the atomic cloud shape between the two probes. However, such attempts lead to marginal improvements in recovered squeezing (see Appendix D). Following the formalism of [15], and as detailed in the Appendix, the loss of homogeneity can be treated as an effective atom loss irrespective of the details of the coupling inhomogeneity: $N_{0} \rightarrow N_{r}$ and $J_{z, 0} \rightarrow J_{z, r}$, where subscript 0 and subscript $r$ stand for observables seen by the near homogeneously coupled state-preparation probe and the inhomogeneously coupled readout probe, respectively.

In earlier squeezed-state measurement-based metrology demonstrations, where the atom-cavity coupling did not substantially change between the state-preparation and readout probes, squeezing efficacy could be directly assessed through comparison of collective spin measurements resulting from back-to-back QND probes. In the current experiment, this approach is no longer accurate. The effective atom numbers and atom-cavity couplings result in different cavity frequency shifts in the two probes. In order to accommodate this change, we translate observed cavity shifts into Bloch vector angles: $\theta_{0}=J_{z, 0} /\left(N_{0} / 2\right)$ and $\theta_{r}=J_{z, r} /\left(N_{r} / 2\right)$ for the preparation and readout probes, respectively (in this experiment $J_{z, 0} \ll N_{0}$ and $J_{z, r} \ll N_{r}$ ). While the measured cavity shifts differ, the mean values for $\theta_{0}$ and $\theta_{r}$ preserve between the two probes (i.e., $\left.\left\langle\theta_{0}\right\rangle=\left\langle\theta_{r}\right\rangle\right)$ (see Appendix A).

\section{RESULTS}

We demonstrate this equivalence experimentally by inserting an additional small microwave rotation between the preparation and readout probes [see Fig. 1(b)] whose phase is $90^{\circ}$ from the atomic phase in order to prepare a Bloch vector angle $\theta_{0}$ away from the equator. We then compare $\left\langle\theta_{r}\right\rangle$ to $\left\langle\theta_{0}\right\rangle$ at different free-fall times. Since $\theta_{0}, \theta_{r} \ll 1$, these angles can be determined experimentally from the ratio of the observed cavity shift to the maximal shift observed when atoms are prepared in the $|\downarrow\rangle$ state. The result is shown in 


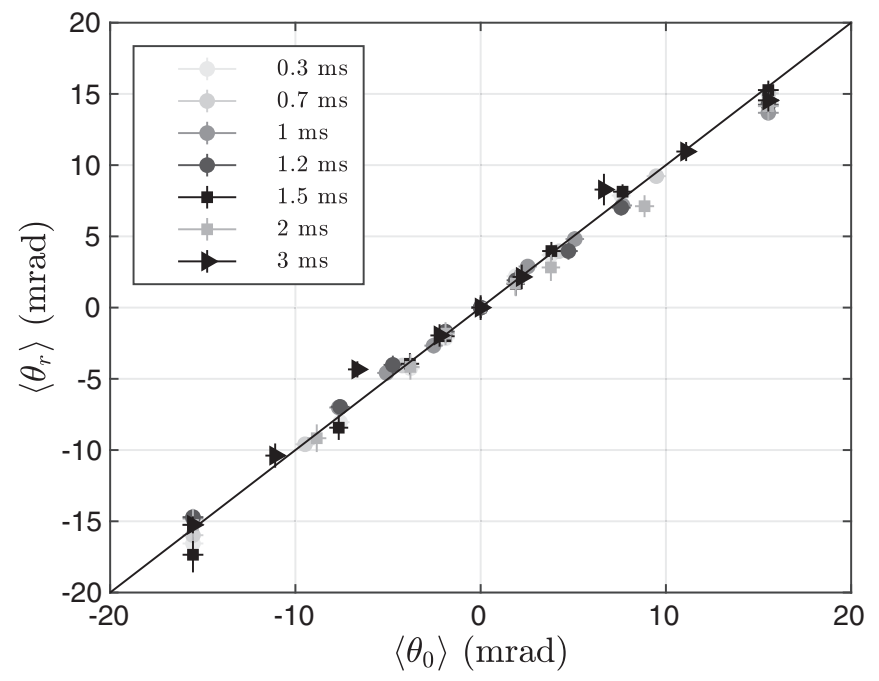

FIG. 2. Relation between $\left\langle\theta_{r}\right\rangle$ and $\left\langle\theta_{0}\right\rangle$ for different free-fall times $\Delta t$. Black line: function $\left\langle\theta_{r}\right\rangle=\left\langle\theta_{0}\right\rangle$. Error bars stand for $68 \%$ confidence interval.

Fig. 2. Linear regression to data points for all the free-fall times with zero intercept gives a slope of $0.97 \pm 0.01(68 \%$ confidence interval), which shows that experimentally we test the angle $\left\langle\theta_{r}\right\rangle$ to be equal to $\left\langle\theta_{0}\right\rangle$ with less than $5 \%$ error. This discrepancy is from the uncertainty in the initial alignment of the microwave phase to the atomic phase. For this data, we use small atom numbers ( $\sim 0000$ atoms) so that the cavity shifts remain well within the linear response of the homodyne cavity readout.

The phase resolution $\Delta \theta$ of the squeezing protocol is determined experimentally from the measured values of $\theta_{r}$ and $\theta_{0}$ over an ensemble of measurements. Specifically, $(\Delta \theta)^{2}=$ $\operatorname{var}\left(\theta_{r}-\theta_{0}\right)$. Figure 3 shows the measured resolution $\Delta \theta$ for ensembles of $N \sim 500000$ spin-squeezed atoms (circles) as a function of free-fall time $\Delta t$ when the state is near the equator of the Bloch sphere. Each data point for $\Delta \theta$ is obtained using more than 700 independent measurements (inset, Fig. 3). The best observed angle sensitivity is 298(8) $\mu \mathrm{rad}$. As expected, $\Delta \theta$ increases with free-fall time since the asymmetry between the two probe couplings increases with this time. For comparison, the projection noise level associated with a coherent spin state with atom number $N_{0}$ measured by the preparation probe is also shown in Fig. 3. $N_{0} \approx N$ is determined from the observed cavity frequency shift, following Ref. [21].

\section{DISCUSSION}

Remarkably, the observed loss in phase resolutionfundamentally associated with the loss in coupling homogeneity of the readout probe-can be accurately described by a model which parametrizes this homogeneity loss in terms of a single parameter, the effective atom number $N_{r}$, regardless of the detailed structure of the coupling. Explicitly, $N_{r} \equiv\left(\sum_{i=1}^{N} \eta_{i}\right)^{2} / \sum_{i=1}^{N} \eta_{i}^{2}$, where $\eta_{i} \in[0,1]$ is the fractional coupling of the $i$ th atom in the ensemble and $N$ is the total atom number. From this definition, it can be shown that

$$
(\Delta \theta)^{2} \simeq \frac{1-\gamma_{r}}{\gamma_{r}} \frac{1}{N_{0}}+\sigma_{p}^{2}+\sigma_{r}^{2},
$$

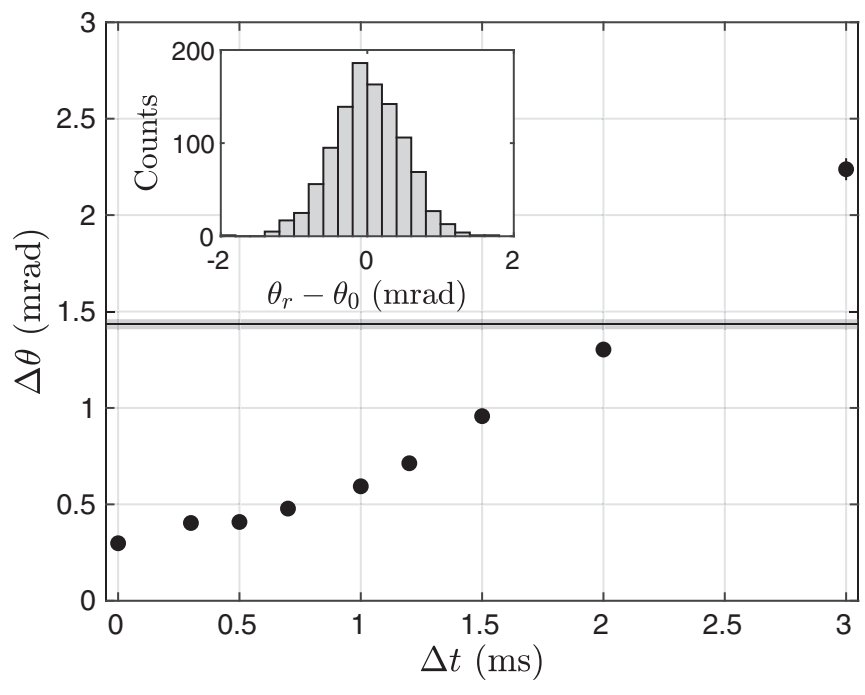

FIG. 3. Single-shot phase sensitivity $\Delta \theta$ as a function of freefall time $\Delta t . \Delta t=0$ indicates no release. Circles show $\Delta \theta$ for spin squeezed state. The solid line is the quantum projection noise level given by atom number $N_{0}$. The width of the shaded region is given by the uncertainty in determination of $N_{0}(68 \%$ confidence interval). Some of the error bars are smaller than the plotted data points. Inset: histogram of $\theta_{r}-\theta_{0}$ at $\Delta t=0.7 \mathrm{~ms}$.

where $\gamma_{r} \equiv N_{r} / N_{0}$ is the ratio of the effective atom number measured by the inhomogeneous readout probe to the atom number measured by the homogeneous preparation probe, and the terms $\sigma_{p}^{2}$ and $\sigma_{r}^{2}$ account for the photon shot noise and spin-flip noise for the preparation and readout probes, respectively. At longer free-fall times, where coupling homogeneity loss plays a significant role $\left(\gamma_{r}\right.$ is small), the $\sigma_{p}$ and $\sigma_{r}$ terms are dominated by the first term in Eq. (1). In the limit of no homogeneity loss $\left(\gamma_{r} \rightarrow 1\right)$, the expression approaches the noise of the initial spin squeezed state.

Comparison of the data shown in Fig. 3 with Eq. (1) requires experimental determination of $\gamma_{r}$. This can be done by noting that $N_{r}$ is defined such that the projection noise in the corresponding effective spin component $J_{z, r}$ for an initially prepared coherent spin state is $\operatorname{var}\left(J_{z, r}\right)=N_{r} / 4$, while $\left|J_{r}\right|=N_{r} / 2$ [14]. Combining these definitions yields $\operatorname{var}\left(J_{z, r}\right) /\left|J_{r}\right|^{2}=1 / N_{r}$, which allows determination of $N_{r}$ through measurement of the ratio $\operatorname{var}\left(J_{z, r}\right) /\left|J_{r}\right|^{2}$ for coherent states. We experimentally determine the value of this ratio through the ratio of the observed fluctuations in the homodyne signal for a coherent spin state on the equator [proportional to $\operatorname{var}\left(J_{z, r}\right)^{1 / 2}$ ] and the overall cavity shift observed when the atoms are instead prepared in the $|\downarrow\rangle$ state (proportional, with the same constant of proportionality, to $\left|J_{r}\right|$ ). Combined with an initial measurement of $N_{0}, \gamma_{r}$ is thus determined. Figure 4(a) shows the resulting inferred values of $\gamma_{r}$ as a function of free-fall time. Since $\gamma_{r}$ is independent of initial atom number, we determine $\gamma_{r}$ with smaller ensembles of $\sim 100000$ atoms to avoid the influence of microwave rotation noise in the preparation of the initial coherent spin state. Substitution of the measurement of $\gamma_{r}$, together with the known value of $\sigma_{p}^{2}+\sigma_{r}^{2}$ and $N_{0}$, into Eq. (1) leads to Fig. 4(b). 

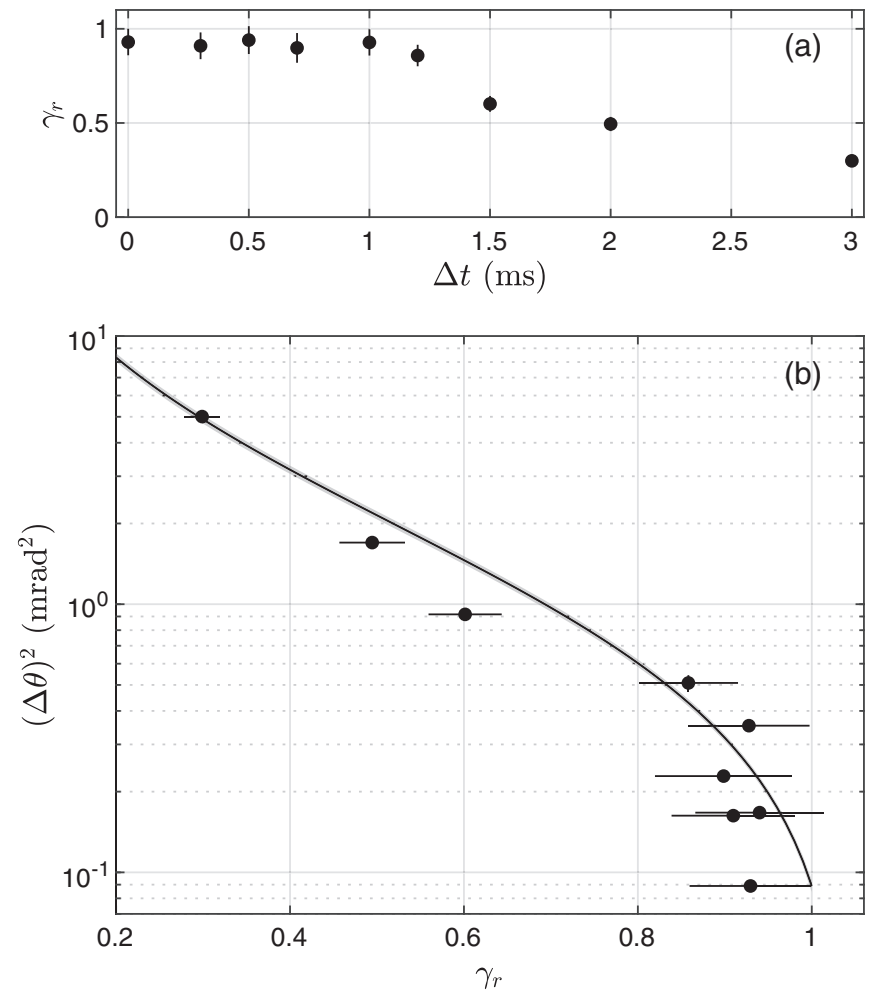

FIG. 4. (a) Measured value of $\gamma_{r}$ as a function of $\Delta t$. (b) $(\Delta \theta)^{2}$ as a function of $\gamma_{r}$. The solid line is a theory curve for $N_{0}=500000$ and $\sqrt{\sigma_{p}^{2}+\sigma_{r}^{2}}=298 \mu \mathrm{rad}$ using Eq. (1). The shaded region is due to the uncertainty in determination of $N_{0}$ and $\sqrt{\sigma_{p}^{2}+\sigma_{r}^{2}}$ (width indicates $68 \%$ confidence interval). Error bars show $68 \%$ confidence interval. Some of the error bars in $y$ axis are smaller than the plotted points.

Metrologically relevant squeezing can be quantified with the Wineland parameter $\xi^{2}[9,22]$, which compares the angle resolution $\Delta \theta$ obtained with a squeezed state to that obtained with an (unsqueezed) coherent spin state having the same number of atoms and also accounts for coherence $C$ of the ensemble. Using the notation defined above, the Wineland parameter $\xi^{2}$ takes the form

$$
\xi^{2}=\left(\frac{\Delta \theta}{1 / \sqrt{N_{r}}} \frac{1}{C}\right)^{2} .
$$

Experimentally, we characterize $C$ with an additional microwave $\pi / 2$ rotation just before recapturing the atoms. We find $C \simeq 0.96$, independent of free-fall time [see Fig. 5(a)]. Figure 5(b) shows the inferred Wineland parameter as a function of $\Delta t$, given the measurements of $\Delta \theta$ and $N_{r}$ with $N \sim 500000$ atoms. Despite substantial loss of homogeneity, metrologically relevant squeezing is observed to persist for time scales as long as $3 \mathrm{~ms}$. We can recover most of the initial squeezing for shorter free-fall times $(\Delta t<1 \mathrm{~ms})$.

\section{CONCLUSION}

Although the successful retrieval of squeezing was limited to $\sim 3 \mathrm{~ms}$ free-fall times in this work, a substantial extension of this duration should be possible using a significantly colder atomic ensemble and an optimized cavity orientation with respect to gravity. We expect the model used to quantify the
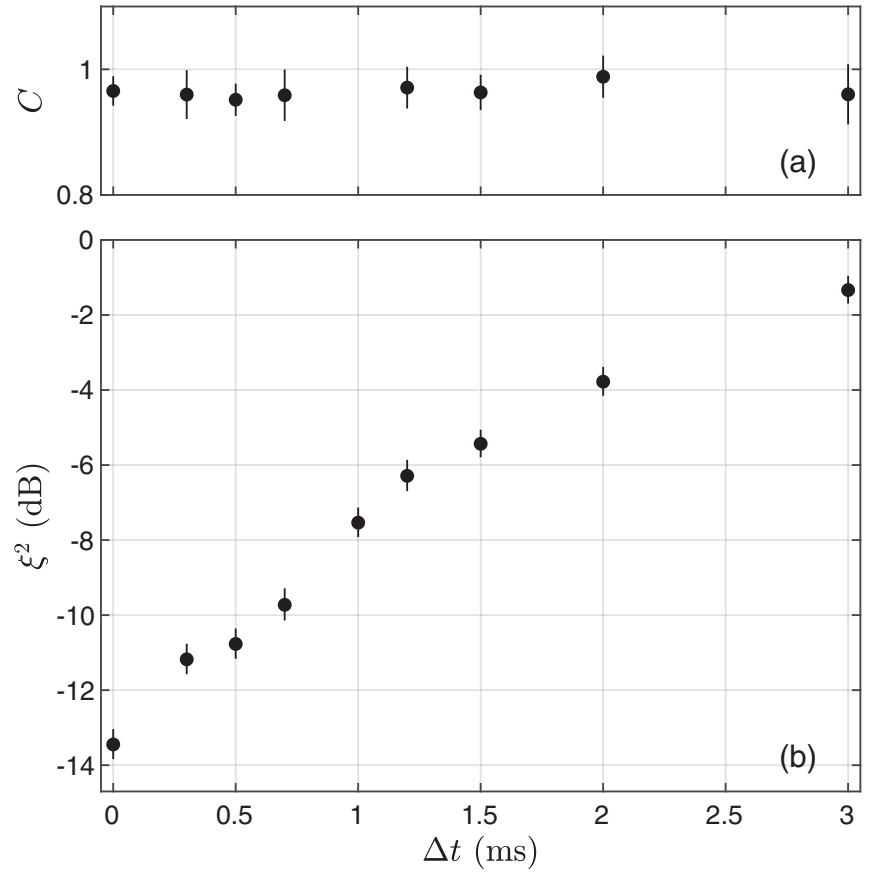

FIG. 5. (a) Coherence of atoms during free falling and (b) retrieved metrologically relevant squeezing $\xi^{2}$ as a function of free-fall time $\Delta t$. Error bars indicate $68 \%$ confidence interval.

loss of squeezing to be useful in designing and predicting the performance of future squeezed-state sensors especially for those that require high bandwidth readout.

\section{ACKNOWLEDGMENTS}

We thank N. Engelsen for comments on the manuscript. This work was supported by the Office of Naval Research, Vannevar Bush Faculty Fellowship, Department of Energy, and Defense Threat Reduction Agency. R.K. was partly supported by the AQT/INQNET program at Caltech.

\section{APPENDIX A: THEORY ON HOMOGENEITY LOSS}

\section{Modeling the loss in atom-cavity coupling homogeneity}

We use $J_{z, 0}$ and $N_{0}$ to stand for collective $J_{z}$ and atom number measured by the homogeneous preparation probe. When the particles are identically prepared and uncorrelated, $\left\langle J_{z, 0}^{\max }\right\rangle=N_{0} / 2$ and $\operatorname{var}\left(J_{z, 0}\right)=N_{0} / 4$. Following the formalism described in [15], we introduce effective observable $J_{z, r}$ and effective atom number $N_{r}$ to model the quantities measured by the inhomogeneous readout probe so that the standard relations for uncorrelated particles $\left\langle J_{z, r}^{\max }\right\rangle=N_{r} / 2$ and $\operatorname{var}\left(J_{z, r}\right)=N_{r} / 4$ are satisfied. Here, $J_{z, r}^{\max }$ is shorthand for $J_{z, r}$ when the quantum state gives $j_{z}^{(i)}=$ $1 / 2$ for all $i$. This state is represented by the Bloch vector that is pointing to the north pole of the Bloch sphere. Accordingly, we write $J_{z, r}=\langle\eta\rangle_{e} \sum_{i} \eta_{i} j_{z}^{(i)} /\left\langle\eta^{2}\right\rangle_{e}=$ $N_{r} \sum_{i} \eta_{i} j_{z}^{(i)} / N_{0}\langle\eta\rangle_{e}$ and $N_{r}=N_{0}\langle\eta\rangle_{e}^{2} /\left\langle\eta^{2}\right\rangle_{e}$, where $\eta_{i} \in[0,1]$ is the fractional coupling of the $i$ th atom and $N_{0}$ is the actual total atom number. Here $\langle\cdot\rangle_{e}$ is the ensemble average of a quantity over the atoms, e.g., $\langle\eta\rangle_{e}=\sum_{i} \eta_{i} / N_{0}$. Given 
the total cavity frequency shift $\delta_{\text {cav }}=\sum_{i} \delta_{0} \eta_{i} j_{z}^{(i)}=\delta_{r} J_{z, r}$, the frequency shift per spin flip seen by the readout probe can be defined as $\delta_{r}=\delta_{0}\left\langle\eta^{2}\right\rangle_{e} /\langle\eta\rangle_{e}$, where $\delta_{0}$ is the frequency shift per spin flip seen by the preparation probe.

Now we relate the observations of $J_{z, r}$ to those of $J_{z, 0}$. We rely on the fact that $\left\langle j_{z}^{(i)}\right\rangle$ and $\left\langle j_{z}^{(i)} j_{z}^{(i \neq k)}\right\rangle$ are independent of $i$ and $k$ owing to the homogeneity of the prepared states. Thus we get

$$
\begin{aligned}
\left\langle J_{z, 0}\right\rangle & =\sum_{i}\left\langle j_{z}^{(i)}\right\rangle=N_{0}\left\langle j_{z}^{(i)}\right\rangle, \\
\left\langle J_{z, 0}^{2}\right\rangle & =\sum_{i, k}\left\langle j_{z}^{(i)} j_{z}^{(k)}\right\rangle \\
& =\sum_{i \neq k}\left\langle j_{z}^{(i)} j_{z}^{(k)}\right\rangle+\sum_{i}\left\langle j_{z}^{(i) 2}\right\rangle \\
& =N_{0}\left(N_{0}-1\right)\left\langle j_{z}^{(i)} j_{z}^{(i \neq k)}\right\rangle+N_{0}\left\langle j_{z}^{(i) 2}\right\rangle, \\
\left\langle J_{z, r}\right\rangle & =\frac{\langle\eta\rangle_{e}}{\left\langle\eta^{2}\right\rangle_{e}} \sum_{i} \eta_{i}\left\langle j_{z}^{(i)}\right\rangle=\frac{N_{r}}{N_{0}}\left\langle J_{z, 0}\right\rangle, \\
\left\langle J_{z, r}^{2}\right\rangle & =\frac{\langle\eta\rangle_{e}^{2}}{\left\langle\eta^{2}\right\rangle_{e}^{2}} \sum_{i, k} \eta_{i} \eta_{k}\left\langle j_{z}^{(i)} j_{z}^{(k)}\right\rangle \\
& =\frac{N_{r}}{N_{0}}\left\langle J_{z, 0}^{2}\right\rangle-\frac{N_{r}}{N_{0}}\left(1-\frac{N_{r}}{N_{0}}\right)\left\langle j_{z}^{(i)} j_{z}^{(i \neq k)}\right\rangle N_{0}^{2}, \\
\left\langle J_{z, r} J_{z, 0}\right\rangle & =\frac{N_{r}}{N_{0}}\left\langle J_{z, 0}^{2}\right\rangle .
\end{aligned}
$$

Defining Bloch vector angles $\theta_{0}=J_{z, 0} /\left(N_{0} / 2\right)$ and $\theta_{r}=J_{z, r} /\left(N_{r} / 2\right)$ for the homogeneous and inhomogeneous probes, respectively, we prove that $\left\langle\theta_{r}\right\rangle=\left\langle J_{z, r}\right\rangle /\left(N_{r} / 2\right)=$ $\left\langle J_{z, 0}\right\rangle /\left(N_{0} / 2\right)=\left\langle\theta_{0}\right\rangle$.

Assuming the atomic state is lying close to the equator of the Bloch sphere and each location in space contains only one atom, we obtain $\left\langle j_{z}^{(i)}\right\rangle=\sigma(\sigma \ll 1)$ and $\left\langle j_{z}^{(i) 2}\right\rangle=$ $1 / 4+\sigma^{2}$. Consequently, $\left\langle j_{z}^{(i)} j_{z}^{(i \neq k)}\right\rangle=\left(\xi^{2}-1\right) / 4\left(N_{0}-1\right)+$ $\sigma^{2}$, where the variance of $J_{z, 0}$ with respect to the coherent spin state (CSS) noise is defined as $\operatorname{var}\left(J_{z, 0}\right) /\left(N_{0} / 4\right)=\xi^{2}$. In addition, $\left\langle j_{z}^{(i) 2}\right\rangle-\left\langle j_{z}^{(i)} j_{z}^{(i \neq k)}\right\rangle=\left[1+\left(1-\xi^{2}\right) /\left(N_{0}-1\right)\right] / 4 \approx$ $1 / 4$, where the approximation is valid if the $J_{z}$ noise is close to the CSS noise; for example, for a $J_{z}$ noise $20 \mathrm{~dB}$ above CSS noise $\left(\xi^{2}=100\right)$, the fractional correction is only $2 \times 10^{-4}$ for 500000 atoms. Therefore,

$$
\begin{aligned}
\operatorname{var}\left(J_{z, r}\right)= & \frac{N_{r}^{2}}{N_{0}^{2}} \operatorname{var}\left(J_{z, 0}\right) \\
& +N_{r}\left(1-\frac{N_{r}}{N_{0}}\right)\left(\left\langle j_{z}^{(i) 2}\right\rangle-\left\langle j_{z}^{(i)} j_{z}^{(i \neq k)}\right\rangle\right) \\
\approx & \gamma_{r}^{2} \operatorname{var}\left(J_{z, 0}\right)+\left(1-\gamma_{r}\right) \frac{N_{r}}{4}, \\
\operatorname{var}\left(J_{z, r}-J_{z, 0}\right) \approx & \left(1-\gamma_{r}\right)^{2} \operatorname{var}\left(J_{z, 0}\right)+\left(1-\gamma_{r}\right) \frac{N_{r}}{4},
\end{aligned}
$$

where $\gamma_{r}=N_{r} / N_{0}$ is the ratio of the effective atom number measured by the inhomogeneous readout probe to the atom number measured by the homogeneous preparation probe.

\section{Modeling back-to-back conditional measurement}

Since $J_{z}$ is inferred from the $X$ quadrature of a probe field through a calibrated discriminator, we label the $X$ quadrature of the two probes in the back-to-back measurements as $X_{p}^{\prime}$ and $X_{r}^{\prime}$, where $p$ and $r$ stand for preparation and readout, respectively. Since these two field modes are uncorrelated, $\operatorname{cov}\left(X_{p}^{\prime}, X_{r}^{\prime}\right)=0$. We also label the collective spin operators for the atoms during the preparation and readout measurements as $J_{z, 0}^{\prime}$ and $J_{z, r}^{\prime}$, respectively. Since before sending probes to the atoms, there are no correlations between the spins and the fields, i.e., $\operatorname{cov}\left(J_{z, r}^{\prime}, X_{p}^{\prime}\right)=\operatorname{cov}\left(J_{z, 0}^{\prime}, X_{p}^{\prime}\right)=$ $\operatorname{cov}\left(J_{z, r}^{\prime}, X_{r}^{\prime}\right)=\operatorname{cov}\left(J_{z, 0}^{\prime}, X_{r}^{\prime}\right)=0$, after probe interactions, the quadrature operators (in the Heisenberg picture) become

$$
\begin{gathered}
X_{p}^{\prime} \rightarrow X_{p}=D_{p} J_{z, 0}^{\prime}+X_{p}^{\prime}, \\
X_{r}^{\prime} \rightarrow X_{r}=D_{r} J_{z, r}^{\prime}+X_{r}^{\prime},
\end{gathered}
$$

where $D_{i}$ are calibrated discriminators determined by the strength of the probes. Thus the inferred $J_{z}$ values from the two probes are $J_{z, 0}=X_{p} / D_{p}$ and $J_{z, r}=X_{r} / D_{r}$. The variance of the inferred $J_{z}$ difference is

$$
\begin{aligned}
& \operatorname{var}\left(J_{z, 0}-J_{z, r}\right) \\
& \quad=\operatorname{var}\left(J_{z, r}^{\prime}-J_{z, 0}^{\prime}\right)+\frac{1}{D_{p}^{2}} \operatorname{var}\left(X_{p}^{\prime}\right)+\frac{1}{D_{r}^{2}} \operatorname{var}\left(X_{r}^{\prime}\right) \\
& \quad \approx\left(1-\gamma_{r}\right) \frac{N_{r}}{4}+\left(1-\gamma_{r}\right)^{2} \operatorname{var}\left(J_{z, 0}^{\prime}\right)+\sigma_{X p}^{2}+\sigma_{X r}^{2} .
\end{aligned}
$$

Here $\sigma_{X p}^{2}$ and $\sigma_{X r}^{2}$ are the squares of $J_{z}$ resolutions by the preparation and the readout probes respectively due to optical shot noise and spin flips. $J_{z, r}^{\prime}$ and $J_{z, 0}^{\prime}$ are $J_{z}$ observables that are defined in Sec. A 1 of the Appendix without the prime. $J_{z, r}$ and $J_{z, 0}$ are inferred quantities through cavity frequency measurements used in the main paper. The second term is an excess noise due to changes in $J_{z}$ between the two probes whose value depends on the outcome of the preparation probe which itself is random.

To eliminate this noise, we work with Bloch vector angle $\theta$. The angles inferred from the $J_{z}$ measurements are defined as $\theta_{0}=J_{z, 0} /\left(N_{0} / 2\right)=2 X_{p} / N_{0} D_{p}$ and $\theta_{r}=$ $J_{z, r} /\left(N_{r} / 2\right)=2 X_{r} / N_{r} D_{r}$, respectively. The variance of the difference between the two inferred angles is

$$
\begin{aligned}
\operatorname{var} & \left(\theta_{r}-\theta_{0}\right) \\
& =\operatorname{var}\left(\theta_{r}^{\prime}-\theta_{0}^{\prime}\right)+\frac{1}{D_{p}^{2} N_{0}^{2} / 4} \operatorname{var}\left(X_{p}^{\prime}\right)+\frac{1}{D_{r}^{2} N_{r}^{2} / 4} \operatorname{var}\left(X_{r}^{\prime}\right) \\
& \approx \frac{1-\gamma_{r}}{N_{r}}+\sigma_{p}^{2}+\sigma_{r}^{2} \\
& =\frac{1-\gamma_{r}}{\gamma_{r}} \frac{1}{N_{0}}+\sigma_{p}^{2}+\sigma_{r}^{2} .
\end{aligned}
$$

Here $\theta_{r}^{\prime}=J_{z, r}^{\prime} /\left(N_{r} / 2\right)$ and $\theta_{0}^{\prime}=J_{z, 0}^{\prime} /\left(N_{0} / 2\right)$. This equation gives the angle resolution for our setup that accounts for the noise due to changes in atom-cavity coupling between the preparation and readout probes $\left(1-\gamma_{r}\right) / \gamma_{r} N_{0}$ and noise from the initial squeezing $\sigma_{p}^{2}+\sigma_{r}^{2}$. 


\section{APPENDIX B: CAVITY READOUT}

We measure resonance frequency shift of a high finesse optical cavity to infer the atomic state and calibrate the atom number in this experiment.

\section{Linewidth broadening}

The cavity frequency shift is measured by comparing the time-dependent homodyne signal to a normalized template taken in the absence of atoms. Corrections to nonlinearities of the cavity frequency response and linewidth broadening factor $\kappa_{s}$ due to atomic scattering are applied in the same way as in Refs. [6,23]. However, the atom-cavity coupling is less homogeneous for the second probe after release recapture; this effect changes the linewidth broadening factor to $\langle\eta\rangle_{e} \kappa_{s}$.

\section{Measurement of maximum cavity frequency shift and $\langle\eta\rangle_{e}$}

To measure maximum cavity frequency shift and $\langle\eta\rangle_{e}$, we prepare all the atoms in $|\downarrow\rangle$ state. Since $500000|\downarrow\rangle$ state atoms give a cavity resonant frequency shift of more than $1 \mathrm{MHz}$, which is far more than the cavity linewidth $\sim 10 \mathrm{KHz}$, we use a different method to measure cavity frequency shift than the back-to-back method used in the main paper [6]. In this new method, the $780 \mathrm{~nm}$ probe is on continuously through the release-recapture (RR) sequence and its frequency is scanned from $50 \mathrm{KHz}$ to $-50 \mathrm{KHz}$ detuned from cavity resonance in $200 \mu$ s that overlaps with the time when the second probe is on during the RR sequence in the back-to-back method. This scan gives a dispersive signal by the homodyne detection when no atom is loaded. This dispersive signal changes its shape due to the added $|\downarrow\rangle$ atoms. Adjusting the starting frequency of the frequency scan brings the dispersive signal back to empty cavity shape, though the frequency range of the scan remains $100 \mathrm{KHz}$. The amount of adjusted frequency tells the maximum cavity frequency shift caused by the amount of added atoms which is counted by fluorescent imaging.

Due to the bandwidth of the laser frequency lock, the maximum adjustment on the frequency scan starting point is limited to $\sim 900 \mathrm{KHz}$. This also limits the maximum added atom number to $\sim 300000$. To get the maximum cavity frequency shift for all different atom numbers $(\sim 50000$, 100000 , and 500000 in this work) and reduce uncertainties, we measure this shift as a function of atom number and fit this function with a straight line (as expected). The maximum cavity frequency shift that corresponds to a specific atom number is found by interpolation or extrapolation.

We measure and linearly fit this frequency shift as a function of atom number for each free-fall time. The slope of each fitting is proportional to $\langle\eta\rangle_{e}$ with the same constant of proportionality. This constant can be calculated by fitting of the zero free-fall time data whose $\langle\eta\rangle_{e}=0.9254$ is known [6]. $\langle\eta\rangle_{e}$ for other free-fall times are thus calculated.

\section{APPENDIX C: COHERENCE MEASUREMENT}

The coherence of the atomic state is measured by microwave-induced Ramsey oscillations. The first Ramsey $\pi / 2$ pulse is the composite $\pi / 2$ microwave pulse at the

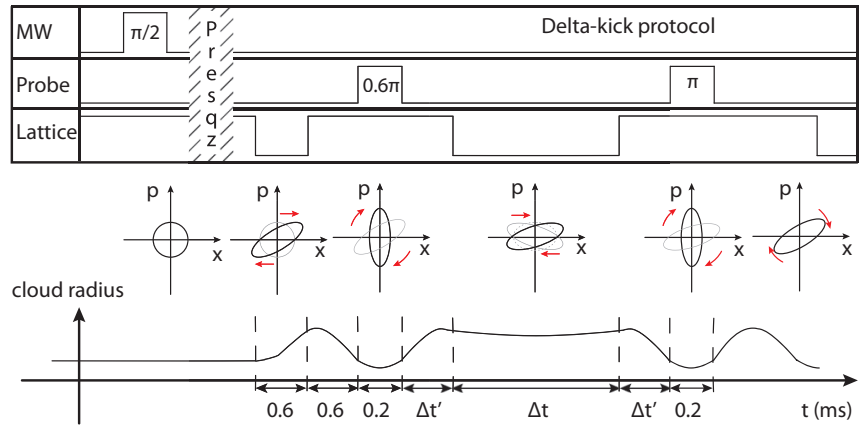

FIG. 6. Delta-kick protocol: timing sequence; illustration for phase-space evolution of the atomic cloud in the transverse direction of the cavity; illustration for rms transverse cloud size evolution. In the phase-space illustration, clouds are modeled to execute harmonic oscillations when trapped in the lattice. MW: microwaves; Presqz: presqueezing by one-axis twisting; $\pi / 2$ : composite microwave pulse that prepares the $50-50$ superposition state; $0.6 \pi$ and $\pi$ : probe power expressed in terms of the ac Stark phase shifts induced between the two atomic states; $\Delta t^{\prime}$ : short free-fall time for atomic phase space reshaping; $\Delta t$ : free-fall time. The values for $\Delta t^{\prime}$ and $\Delta t$ are shown in Table I.

beginning of the experimental sequence to prepare the initial superposition state where the atoms are trapped by the lattice. The second $\pi / 2$ pulse is applied after atoms have freely fallen for a certain amount of time. The phase of this pulse is adjusted to be roughly $90^{\circ}$ to the atoms by a frequency shift key and is scanned by a small amount. This scan covers the bottom (top) part of the Ramsey fringes. A quadratic fit is applied to this part of the fringes. The lowest (highest) point of this fit stands for the coherence. The $68 \%$ prediction interval of that point gives the uncertainty on the coherence measurement. Every step in the sequence before the release remains the same as the squeezing measurement. Since the cavity resonance is shifted out of the cavity linewidth, a fluorescence population spectroscopy is used to measure the collective $J_{z}$ [21]. This coherence is measured with $\sim 500000$ atoms.

\section{APPENDIX D: DELTA-KICK PROTOCOL}

In the "delta-kick" protocol (Fig. 6), we utilize a series of lattice on-off sequences to reshape the phase-space distribution of the atomic cloud. The timing in this protocol is based on a simulation assuming the lattice is a harmonic trap and is experimentally chosen. See Table I. This protocol gives less

TABLE I. Delta-kick protocol timing sequence. $\Delta t^{\prime}$ stands for the short free-fall time before the measurement sequence to reshape the phase-space distribution of the cloud. $\Delta t$ is the free-fall time that is used for free-space atomic sensors.

\begin{tabular}{lc}
\hline \hline$\Delta t^{\prime}(\mathrm{ms})$ & $\Delta t(\mathrm{~ms})$ \\
\hline 0.8 & 0.4 \\
0.7 & 0.7 \\
0.6 & 1.2 \\
0.5 & $1.4,2.0,3.0,4.0,5.0,6.0,7.0$ \\
\hline \hline
\end{tabular}


coherence $(<92 \%)$ compared to the RR protocol irrespective of free-fall times, because the extra lattice on-off sequences induce more inhomogeneous ac Stark shifts on the atoms. The metrologically relevant squeezing decreases to 0 in less than $5 \mathrm{~ms}$ free-fall times.

\section{APPENDIX E: UNCERTAINTY CALCULATIONS}

We calculate uncertainties using a standard error propagation formula where the uncertainty of each quantity stands for $68 \%$ confidence interval. These quantities are assumed to be independent and their errors are assumed to follow normal distributions. When determining the uncertainties of $\Delta \theta$, only statistical uncertainty on cavity frequency measurements is considered. This uncertainty is estimated by a bootstrapping method where we resample 10000 times from the measured frequency distribution, calculate 10000 standard deviations from the resampled data, and estimate this uncertainty as the standard deviation of these 10000 standard deviations, whereas the uncertainty of squeezing includes uncertainties on cavity frequency and atom number measurements.
[1] G. Rosi, F. Sorrentino, L. Cacciapuoti, M. Prevedelli, and G. M. Tino, Nature (London) 510, 518 (2014).

[2] F. Sorrentino, Q. Bodart, L. Cacciapuoti, Y.-H. Lien, M. Prevedelli, G. Rosi, L. Salvi, and G. M. Tino, Phys. Rev. A 89, 023607 (2014).

[3] B. J. Bloom, T. L. Nicholson, J. R. Williams, S. L. Campbell, M. Bishof, X. Zhang, W. Zhang, S. L. Bromley, and J. Ye, Nature (London) 506, 71 (2014).

[4] N. Hinkley, J. A. Sherman, N. B. Phillips, M. Schioppo, N. D. Lemke, K. Beloy, M. Pizzocaro, C. W. Oates, and A. D. Ludlow, Science 341, 1215 (2013).

[5] M. Kitagawa and M. Ueda, Phys. Rev. A 47, 5138 (1993).

[6] O. Hosten, N. J. Engelsen, R. Krishnakumar, and M. A. Kasevich, Nature (London) 529, 505 (2016).

[7] R. J. Sewell, M. Koschorreck, M. Napolitano, B. Dubost, N. Behbood, and M. W. Mitchell, Phys. Rev. Lett. 109, 253605 (2012).

[8] W. Muessel, H. Strobel, D. Linnemann, D. B. Hume, and M. K. Oberthaler, Phys. Rev. Lett. 113, 103004 (2014).

[9] M. H. Schleier-Smith, I. D. Leroux, and V. Vuletic, Phys. Rev. Lett. 104, 073604 (2010).

[10] R. Schmied, J.-D. Bancal, B. Allard, M. Fadel, V. Scarani, P. Treutlein, and N. Sangouard, Science 352, 441 (2016).

[11] K. Maussang, G. E. Marti, T. Schneider, P. Treutlein, Y. Li, A. Sinatra, R. Long, J. Estève, and J. Reichel, Phys. Rev. Lett. 105, 080403 (2010).
[12] J. Estève, C. Gross, A. Weller, S. Giovanazzi, and M. K. Oberthaler, Nature (London) 455, 1216 (2008).

[13] J. Appel, P. J. Windpassinger, D. Oblak, U. B. Hoff, N. Kjærgaard, and E. S. Polzik, Proc. Natl. Acad. Sci. USA 106, 10960 (2009).

[14] K. C. Cox, G. P. Greve, J. M. Weiner, and J. K. Thompson, Phys. Rev. Lett. 116, 093602 (2016).

[15] J. Hu, W. Chen, Z. Vendeiro, H. Zhang, and V. Vuletić, Phys. Rev. A 92, 063816 (2015).

[16] K. C. Cox, G. P. Greve, B. Wu, and J. K. Thompson, Phys. Rev. A 94, 061601(R) (2016).

[17] Z. Chen, J. G. Bohnet, S. R. Sankar, J. Dai, and J. K. Thompson, Phys. Rev. Lett. 106, 133601 (2011).

[18] J. Lee, G. Vrijsen, I. Teper, O. Hosten, and M. A. Kasevich, Opt. Lett. 39, 4005 (2014).

[19] A. Kuzmich, N. P. Bigelow, and L. Mandel, Europhys. Lett. 42, 481 (1998).

[20] I. D. Leroux, M. H. Schleier-Smith, and V. Vuletić, Phys. Rev. Lett. 104, 073602 (2010).

[21] O. Hosten, R. Krishnakumar, N. J. Engelsen, and M. A. Kasevich, Science 352, 1552 (2016).

[22] D. J. Wineland, J. J. Bollinger, W. M. Itano, F. L. Moore, and D. J. Heinzen, Phys. Rev. A 46, R6797 (1992).

[23] N. J. Engelsen, Ph.D. thesis, Stanford University, 2016. 